\title{
Les filles sont oubliées : rediriger les interventions en matière de VIH vers les plus vulnérables
}

Judith Bruce

Population Council

Follow this and additional works at: https://knowledgecommons.popcouncil.org/departments_sbsr-pgy

Part of the Demography, Population, and Ecology Commons, Family, Life Course, and Society

Commons, Gender and Sexuality Commons, and the International Public Health Commons

How does access to this work benefit you? Let us know!

\section{Recommended Citation}

Bruce, Judith. 2007. "Les filles sont oubliées : rediriger les interventions en matière de VIH vers les plus vulnérables," Promouvoir la santé, la sécurité et la productivité transitions vers l'âge adulte Document no. 23. New York: Population Council. 


\section{Les filles sont oubliées : rediriger les interventions en matière de VIH vers les plus vulnérables}

\section{Préparé par Judith Bruce}

A lors que nous entrons dans la troisième décennie de la pandémie de VIH en Afrique subsaharienne, les jeunes femmes, le plus souvent les adolescentes, sont le nouveau visage de la maladie. Le VIH/SIDA menace en particulier les jeunes femmes et les adolescentes qui se trouvent dans des situations sociaux-économiques peu favorables, celles qui ne peuvent éviter, gérer ni échapper à des relations sexuelles à risque au sein même du mariage ou hors mariage.

Les jeunes filles déscolarisées, en particulier âgées de 10 à 14 ans et vivant seules, sont les plus vulnérables. Les jeunes filles qui viennent d'emménager en zone urbaine, surtout celles qui sont domestiques, qui s'occupent de familles infectées par le VIH ou qui sont chefs de famille sont peut-être soumises à une forte pression pour subvenir à leurs besoins comme à ceux de leurs parents, frères ou sœurs. Les filles mariées sont sujettes à des relations sexuelles fréquentes, non protégées, souvent avec des partenaires plus âgés, et peuvent de ce fait, courir un risque plus élevé de contracter le VIH.

Les filles présentant un risque d'être exploitées sexuellement ne représentent pas une petite minorité : on peut les compter par centaines de millions. Et, malgré leur grand nombre, les interventions existantes ne parviennent pas à atteindre la plupart d'entre elles. De fait, la majorité des programmes pour les compétences nécessaires à la vie courante, de développement social et de santé pour les jeunes ne réussissent pas à atteindre les plus vulnérables qui se trouvent sur le chemin du VIH.

\section{Déterminants structurels du risque}

La vulnérabilité des adolescentes face au VIH, en particulier en Afrique subsaharienne, prend ses racines au sein des conditions structurelles clés suivantes : (1) isolement social, (2) non-scolarisation pendant l'adolescence, (3) mariage des enfants, (4) acti- vité sexuelle à risque et (5) la pression pour travailler et obtenir un revenu.

\section{L'isolement social}

Un obstacle structurel majeur pour les jeunes filles et les jeunes femmes est leur degré d'isolement social. Dans le contexte de la pandémie de $\mathrm{VIH}$, cet isolement a d'énormes conséquences. Par exemple, les jeunes filles les plus isolées socialement dans le Kwazulu-Natal en Afrique du sud, ont six fois plus de chance d'avoir eu des rapports sexuels forcés que la plupart de jeunes filles qui sont socialement connectées (Hallman et Diers 2004). L'isolement social, la pauvreté, le statut d'orphelin et le statut d'écolier ont été, de manière indépendante, sont liés aux rapports sexuels forcés ou contre échange de cadeaux, argent, abri et nourriture (Hallman 2004 et 2005; Hallman et Diers 2004). Parmi les femmes de 18 à 24 ans du KwaZulu-Natal, posséder un statut social plus élevé est lié à une plus grande exposition aux messages sur le VIH dans les médias et elles ont plus de chance de faire un test VIH (Hallman et al. 2007).

Quelle que soit l'appartenance à un groupe de revenu, les filles possèdent systématiquement un réseau d'amies plus restreint et moins fiable que les garçons et il existe un moindre nombre d'espaces sûrs et de soutien où elles peuvent retrouver leurs amies (Erulkar et al. 2004b; Hallman et Diers 2004). Une étude récente menée à Kibera au Kenya (une communauté de 800000 habitants et également site du plus grand bidonville d'Afrique) a montré que seulement un quart des 90000 adolescentes âgés de 10 à 19 ans avaient accès à un endroit sûr pour se retrouver entre elles en dehors de la maison et de l'école (Erulkar et Matheka 2007). Une étude participative y étant associée, conduite par des jeunes filles leaders a montré que moins d'un pour cent des filles ont accès une fois par semaine à un programme 
social dans un espace réservé aux filles. L'accès insignifiant à un espace réservé aux filles représente une perte émotionnelle mais il leur dénie également l'accès aux programmes qui pourraient les informer, les aider à acquérir des compétences et leur offrir des services directement ou sur recommandation.

\section{La déscolarisation}

Les filles jeunes et déscolarisées font face à des menaces importantes concernant leur santé et leur sécurité allant des conditions de travail forcé dans les zones urbaines au mariage précoce en zones rurales. Pour beaucoup, quitter des conditions misérables et un mariage dangereux pour un avenir incertain en ville constitue un phénomène dangereux et apparenté (Erulkar et al. 2004a and 2004b).

Une étude menée au niveau mondial sur les inscriptions à l'école a révélé que les adolescentes de 15 à 17 ans non mariées et scolarisées étaient moins susceptibles d'avoir eu des rapports sexuels que les filles non scolarisées ; leur taux de grossesse non désirée ou de infections sexuellement transmissibles est aussi moins élevé (Lloyd 2005).

\section{Le mariage des enfants}

Si rien ne change, au cours des dix prochaines années, 100 millions de filles seront mariées alors qu'elles sont encore mineures. La santé sexuelle des filles est à plus grand risque chez les filles mariées que chez les jeunes filles non mariées. Les filles mariées ont généralement des partenaires plus âgés que les filles du même âge ayant une vie sexuelle active. Elles doivent faire face à une pression intense pour tomber enceinte avant que leur corps ne soit prêt et ont des rapports sexuels plus fréquemment que leurs pairs sexuellement actives (Clark 2004; Clark, Bruce et Dude 2006). Les adolescentes mariées ont tendance à avoir un réseau limité d'amies, si elles en ont, une mobilité sociale restreinte et un niveau d'éducation peu élevé ainsi qu'un accès limité aux médias modernes et aux messages de santé (Erulkar et al. 2004b).

\section{Une activité sexuelle dans des conditions dangereuses}

L'exclusion effective des jeunes filles des efforts de prévention du VIH a été favorisée par l'ignorance et le déni évident des conditions s'appliquant aux rapports sexuels pendant l'adolescence. Une grande proportion de ces rapports se déroule à l'intérieur même du mariage et les filles mariées indiquent souvent que leur initiation sexuelle fut un acte forcé ainsi que les rapports suivants (Erulkar et al. 2004b). Parmi les filles non mariées, l'initiation sexuelle relève aussi souvent d'une coercition. La moitié des filles les plus pauvres âgées de 14 à 24 ans dans la métropole de Durban au Kwazulu-Natal rapporte que leur premier rapport sexuel a été forcé (Hallman 2005). Ces jeunes filles ne font pas partie des groupes à risque traditionnels tels que les drogués ou les travailleurs de l'industrie du sexe.

\section{Grandir seule et dans le dénuement total}

L'adolescence est un moment critique pour beaucoup de filles quand les vulnérabilités sociales et économiques sont accompagnées de menaces sérieuses envers leur santé. Dans la plupart des pays, la transition de l'école primaire à l'école secondaire se fait pendant la pré-adolescence. De nombreuses familles attendent des filles qu'elles quittent l'école et commencent à travailler pour subvenir et contribuer aux besoins de la famille. Celles qui restent scolarisées pendant cette période peuvent être confrontées à des menaces concernant leur sécurité et leur respectabilité pour les encourager à quitter l'école.

L'absence de l'un ou des deux parents - en particulier au début de l'adolescence - accroît la vulnérabilité des filles. Dans de nombreux pays subsahariens, les filles âgées de 10 à 14 ans vivent avec un seul parent ou seule (Chong, Hallman et Brady 2006). Des conditions encore plus extrêmes de vie existent parmi les enfants vivants sans parents et non scolarisés, ce qui est le cas de $8 \%$ des filles en zone urbaine parmi les pays d'Afrique subsaharienne dans lesquels la DHS a été conduite depuis 2000 ; cette proportion est encore plus élevée dans certains pays, jusqu'à $18 \%$ au Burkina Faso et $21 \%$ au Mali. Sans rien, sans compétences ou protection, ces jeunes filles sont les proies de l'exploitation sexuelle et du travail forcé.

\section{L'impossibilité d'atteindre les plus vulnérables}

Les populations en zones rurales et non scolarisées, les pré-adolescents (particulièrement les filles) vivant sans leurs parents et les filles mariées ont très peu ou pas du tout accès à un large éventail de programmes pour les jeunes. Dans un cas classique d'inversion des avantages, les adolescents possédant le plus ont accès au plus grand nombre de ressources et ceux ayant le moins et qui font face aux plus grands risques sont ceux qui reçoivent le moins.

Une étude récente conduite à Addis-Abeba en Éthiopie, où $30 \%$ des filles de 10 à 14 ans vivent sans parents, montre que seulement $0,5 \%$ de tous les programmes était mis à disposition de ces jeunes filles (Mekbib, Erulkar et Belete 2005). La situation éthiopienne n'est pas unique. Des faits similaires sont observés au Burkina Faso, en Guinée Bissau et en Mauritanie. Une étude conduite au Burkina Faso a montré que seulement $19 \%$ de la population bénéficiant d'un centre pour jeunes étaient des filles (les plus jeunes, âgées de 10 à 14 ne représentaient que $3 \%$ ), alors que $36 \%$ des « jeunes » étaient des hommes de plus de 20 ans (Lardoux 2006 en français).

Les filles mariées, qui participent rarement aux clubs, organisations ou programmes, ont également un accès limité aux mes- 
sages médiatiques, une situation potentiellement grave de conséquences puisque la vaste majorité de l'activité sexuelle des filles pendant l'adolescence (94\% en Éthiopie) se déroule à l'intérieur du mariage (Bruce et Clark 2003).

Même lorsque les programmes atteignent les filles mariées, les stratégies de prévention du VIH actuelles (abstinence, réduction de la fréquence des rapports sexuels, utilisation de préservatifs, connaissance du statut VIH de son partenaire et éviter les relations sexuelles avec un partenaire infecté) sont virtuellement impossibles à respecter dans leur cas.

\section{Solutions : avancer avec ce dont on dispose}

II existe des ressources pour remédier à la situation : de bonnes lois peuvent être mises en place, des droits peuvent être accordés, des programmes et dans de nombreux lieux, d'importants réseaux de services sociaux-économiques et de santé et des initiatives de citoyenneté peuvent être réorientés pour atteindre ces jeunes filles et construire leurs compétences de protection.

\section{Localiser les concentrations importantes de jeunes filles vulnérables} Nous devons accomplir un travail plus efficace quant à la localisation et l'identification des jeunes femmes qui sont concentrées dans des communautés avec un fort taux de $\mathrm{VIH}$ en :

- analysant les données réunies par les Demographic and Health Surveys pour localiser les jeunes filles dans les communautés rurales et les quartiers et en les complétant par une évaluation qualitative ;

- soutenant les initiatives de santé destinées aux communautés et aux enfants qui utilisent les contacts de maison en maison pour identifier les filles et les garçons à risque ; et en

- localisant les services où les jeunes filles (et garçons) vulnérables se retrouvent, comme les arrêts de bus.

\section{Assurer la scolarité pendant toute l'adolescence}

S'assurer que les filles arrivent à l'école à l'heure et y restent pendant toute leur adolescence est crucial. Manquer l'école, aussi mauvaise qu'elle puisse être, signifie passer à côté d'importants avantages didactiques, sociaux et de santé (y compris la santé reproductive). Une nouvelle génération de programmes d'enseignement généraliste est prometteuse, même si de nombreux systèmes scolaires fonctionnent à peine.

\section{Retarder et planifier un mariage avec un partenaire plus sûr}

Si les lois qui interdisent le mariage des enfants étaient respectées, les jeunes filles auraient plus de chance de retarder les rapports sexuels jusqu'à ce qu'elles puissent légalement décider de se marier et dans quelles conditions. Reconnaître que le mariage ne représente pas un terrain sexuel sûr est crucial (Erulkar et al. 2004a et 2004b).
Des programmes pilotes assurant la promotion d'une transition saine et sûre vers le mariage doivent voir le jour. Les jeunes femmes mariées lorsqu'elles en ont eu l'opportunité, ont participé avec enthousiasme aux clubs proposant des cours de rattrapage, un apprentissage de la lecture, des compétences de vie, des formations pour acquérir des compétences nécessaires à la vie courante, un soutien pour qu'une grossesse et qu'une naissance se déroulent sans incident ainsi qu'un accès aux tests VIH et aux traitements (Erulkar et al. 2004b).

\section{Espaces réservés aux filles}

Créer des espaces réservés aux filles est une des stratégies clés pour la transformation de leur prise de conscience et l'expansion de leurs connaissances et pour fournir une plate-forme à partir de laquelle on peut proposer des services de santé, directement ou sur recommandation.

Des espaces sûrs peuvent être institutionnalisés à un coût relativement peu élevé lorsqu'ils sont établis dans les endroits publics tels que les centres pour jeunes, les centres communautaires, les écoles ou les institutions volontaires, y compris les organisations religieuses, les églises, les mosquées ou les organisations non-gouvernementales. Celles-ci existent dans presque toutes les communautés et les investissements ont déjà été faits, afin que des sessions spécifiques selon le sexe et l'âge puissent être accessibles aux filles.

\section{Créer des ressources de mentorat est crucial}

Les perspectives de changement pour les filles sont limitées lorsqu'il n'y a pas de modèles à suivre ou de mentors parmi les femmes plus âgées. Les jeunes femmes qui ont un diplôme de fin d'études et qui n'ont pas d'emploi sont des ressources de grande valeur non exploitées. L'expérience montre que même dans les communautés défavorisées, les femmes mentors ayant typiquement entre 17 et 30 ans peuvent être recrutées contre une faible rémunération. Ces jeunes femmes parlent la langue locale et sont enracinées dans la culture et connaissent les codes de la communauté et peuvent être de bons modèles pour les jeunes filles dans le besoin (Binti Pamoja Center 2006 ; Erulkar et al. 2006).

Repenser les programmes de compétences de vie courante pour répondre aux besoins des jeunes filles

Les jeunes filles, quelle que soit leur communauté ou leur situation financière, sont très intéressées par le développement de leurs compétences de vie courante et expriment un intérêt pour les groupes, les plans d'épargne et les connaissances financières qui s'appliquent à leur tranche d'âge et leur contexte. Les lieux où les programmes créés pour les jeunes filles et femmes sont dispensés pourraient être le lieu de réunion de club d'épargne au sein duquel elles pourraient acquérir des connaissances finan- 
cières, accéder à l'épargne et à un travail digne. Les programmes de compétences de vie courante doivent être adaptés à leurs besoins ; les programmes de micro-finance qui ne sont pas suffisamment attentifs aux besoins sociaux des jeunes filles et qui exigent une demande de prêt sans une connaissance financière et en épargne préalable, augmentent la vulnérabilité des jeunes filles (Population Council 2005; Erulkar et al. 2006).

\section{Conclusion : recadrer les opportunités des adolescentes} Le soutien social et les structures pour les adolescentes peuvent être systématiquement modifiés, tout comme grâce à eux, l'évolution de la pandémie. Les jeunes filles sont des partenaires importants mais les familles et les communautés doivent aussi être impliquées. Sinon, les plus jeunes, les plus pauvres des femmes continueront de supporter le fardeau croissant et disproportionné de cette tragédie humaine.

\section{Références bibliographiques et publications similaires}

Binti Pamoja Center. 2006. « Financial literacy education : A curriculum for adolescent girls in Kibera Trainer's Guide » Washington, DC: The Global Financial Education Program.

Bruce, Judith and Shelley Clark. 2003. « Including married adolescents in adolescent reproductive health and HIVIAIDS policy » background paper presented at the WHO/UNFPA/Population Council Technical Consultation on Married Adolescents, Geneva, 9-12 December.

Bruce, Judith and Amy Joyce (eds). Forthcoming. " The girls left behind: The failed reach of current schooling, child health, youthserving, and livelihood programs for girls living in the path of HIV » New York : Population Council.

Chong, Erica, Kelly Hallman, and Martha Brady. 2006. Investing When It Counts : Generating the Evidence Base for Policies and Programs for Very Young Adolescents-Guide and Tool Kit. New York : UNFPA and Population Council.

Clark, Shelley. 2004. « Early marriage and HIV risks in sub-Saharan Africa » Studies in Family Planning 35(3) : 149-160.

Clark, Shelley, Judith Bruce, and Annie Dude. 2006. " Protecting girls from HIVIAIDS : The case against child and adolescent marriage » International Family Planning Perspectives 32(2) : 79-88.

Erulkar, Annabel S. and James K. Matheka. 2007. Adolescents in the Kibera Slums of Nairobi, Kenya. Nairobi : Population Council.

Erulkar, Annabel, Tekle-Ab Mekbib, Negussie Simie, and Tsehai Gulema. 2004a. The Experience of Adolescence in Rural Amhara Region, Ethiopia. Accra, Ghana : Population Council.
- 2004b. Adolescent Life in Low Income and Slum Areas of Addis Ababa, Ethiopia. Accra, Ghana : Population Council.

Erulkar, Annabel S., Judith Bruce, et al. 2006. Tap and Reposition Youth (TRY) : Providing Social Support Savings, and Microcredit Opportunities for Young Women in Areas with High HIV Prevalence. SEEDS No. 23. New York : Population Council.

Hallman, Kelly. 2004. « Socioeconomic Disadvantage and Unsafe Sexual Behaviors Among Young Women and Men in South Africa »Policy Research Division Working Paper No. 190. New York : Population Council.

—. 2005. «Gendered socioeconomic conditions and HIV risk behaviours among young people in South Africa » African Journal of AIDS Research 41(1) : 37-50.

Hallman, Kelly and Judith Diers. 2004. « Social isolation and economic vulnerability : Adolescent HIV and pregnancy risk factors in South Africa » paper presented at the Annual Meeting of the Population Association of America, Boston, 1-3 April.

Hallman, Kelly, Kasthuri Govender, Emmanuel Mbatha, Rob Pattman, Deevia Bhana, and Jill Walsh. 2007. « Social capital, socioeconomic aspirations, and HIV risk behaviors among poor South African youth » paper presented at the Third South African AIDS Conference, Durban, 6 June.

Lardoux, Solène. 2006. «Exercice de couverture des activités de paires éducateurs au Burkina Faso? Rapport final » New York : UNFPA and Population Council.

Lloyd, Cynthia (ed.). 2005. "Schooling and adolescent reproductive behavior in developing countries » in Growing Up Global : The Changing Transitions to Adulthood in Developing Countries. Washington, DC : The National Academies Press.

Mekbib, Tekle-Ab, Annabel Erulkar, and F. Belete. 2005. «Who are the targets of youth programs? Results of a capacity building exercise in Ethiopia » Ethiopian Journal of Health Development 19(1) : 60-62.

Population Council. 2005. « Building assets for safe, productive lives: A report on a workshop on adolescent girls' livelihoods " Compiled by Erica Chong. New York : Population Council.

__ 2006. « How to conduct a coverage exercise : A rapid assessment tool for programs and services » New York : Population Council.

\section{Donateurs}

Dorothy and Lewis Cullman Foundation, UK Department for International Development (DFID), The Dickler Family Foundation, EMpower (The Emerging Markets Foundation), The Ford Foundation, Bill \& Melinda Gates Foundation, Nike Foundation, United Nations Foundation, United Nations Population Fund (UNFPA)

Pour plus d'information ou des copies d'autres lettres d'information, veuillez contacter publications@popcouncil.org Si vous souhaitez consulter des ressources complémentaires, veuillez consulter www.popcouncil.org/pgy

Population Council

One Dag Hammarskjold Plaza

New York, New York 10017 USA

(C) 2007 The Population Council, Inc. 